self-reported. Method of selecting the subject was randomised. Ten anthropometric dimensions were needed to calculate the anthropometric somatotype:: height, weight, chest circumference, arm span, arm length, skinfolds (biceps, triceps, subscapular, supraspinale and calf), two biepicondylar bone breadth (humerus, femur) and two limb girths (arm flexed and tensed calf). By means of equations, anthropometric somatotype was obtained into which data were entered. Results showed that (1) sprint freestyle and backstroke swimmers were taller and heavier, compared with others, for both sexes. (2) Butterfly group swimmer had a distinct physical structure with lowest values for height and weight. (3) Significant correlations were obtained between swimming performance and somatometric variables such as height, weight, chest circumference, arm span, arm length, endomorphy, mesomorphy, ectomorphy and fat percentage. This study also concluded that swimmers in different swimming styles or events have variations in their body size and form at an early age. Early determination of somatotype of swimmers or athletes participating in different sports helps to improve their performance and development of body according to their requirement in particular sports or event.

\title{
40 RELATIONSHIP BETWEEN DIFFERENT SWIMIMING STYLES AND SOMATOTYPE IN NATIONAL LEVEL SWIMMERS
}

Sonia, Shishir Nigam Department of Therapies and Health Sciences, Faridabad Institute of Technology, Manav Rachna Educational Institution, Faridabad, Haryana, India

10.1136/bjsm.2010.078725.40

This study had been done to categorise the swimmers in India according to there somatotype in different swimming styles at an early age. This study aims to find out the somatotype of swimmers in different swimming styles and analyse the relationship between somatometric variables and swimming performance in competitive national swimmers under 19 years of age. Total of 96 swimmers (50 males and 46 females) aged younger than 19 years were picked up on their age-group championships and classification of the swimmers was done according to their primary swimming specialty, as 